\title{
Designing of potential vaccine candidates of fused cathepsin $L$ and tropomyosin genes of Rhipicephalus (Boophilus) annulatus tick larva
}

\author{
Zahra Asadollahi ${ }^{1}$, Sedigheh Nabian ${ }^{1 *}$, Mohammad Taheri $^{2}$, Elahe Ebrahimzadeh ${ }^{1}$, \\ Mohammad Mehdi Ranjbar ${ }^{3}$
}

1. Faculty of Veterinary Medicine, University of Tehran, Tehran, Iran

2. Rastegar Reference Laboratory, Faculty of Veterinary Medicine, University of Tehran, Tehran, Iran

3. Razi Vaccine and Serum Research Institute, Agricultural Research, Education and Extension Organization (AREEO), Karaj, Iran

*Corresponding author:Tel: +98 2161117000 Fax: +98 2166933222

Address: Department of Parasitology, Faculty of Veterinary Medicine, University of Tehran, Gharib Ave,

Tehran, Iran

E-mail: nabian@ut.ac.ir

Received; 2017/12/1 revised; 2018/01/2 accepted; 2018/04/21

\begin{abstract}
Introduction: Regarding emerging tick resistance against acaricides, researches have been shifted toward alternative approaches such as immunologic methods. Vaccine preparation is an alternative way in which choosing appropriate protein with high immune induction potency is a prerequisite. In addition according to studies, using more than one protein could better enhance the immune induction and antibody production. Choosing immunogenic epitopes from selected proteins and adjoining them with a suitable linker is one of the novel approaches in vaccine design.
\end{abstract}

Materials and methods: Based on the fact that both cathepsin and tropomyosin proteins of Rhipicephalus tick were previously recognized as potent immunogenic antigens, we predicted the immunogenic epitopes of these proteins by immunoinformatic methods. Among studied epitopes, those that were met by multiple bioinformatics tools were used.

Results: Finally, the polytopic construction was designed by assembling the selected epitopes and connecting them with linkers.

Conclusion: Using immunoinformatic tools, we predicted the characteristics of two genes of Rhipicephalus annulatus tick larva as fused potent vaccine candidates namely, cathepsin and tropomyosin.

Keywords: Tropomyosin, Cathepsin, Rhipicephalus annulatus, vaccine design, Iran

\section{Introduction}

The cattle tick, Rhipicephalus (Boophilus) annulatus (Family Ixodidae), is one of the most abundant tick species worldwide specifically in tropical and subtropical territories (1). This species is mainly found in North of Iran (2). Cattle is the principal host for this obligate ectoparasite, but it has also been found occasionally in horse and deer. Rhipicephalus is considered as a potent vector for some bacterial and parasitic pathogens such as Anaplasma and Babesia (3). Additionally, tick infestation usually provokes a notable financial burden in livestock industry through blood

Copyright (C) 2018 Journal of Basic Research in Medical Science. This is an open access article distributed under the terms of the Creative Commons Attribution international 4.0 International License (https://creativecommons.org/licenses/by/4.0/) which permits copy and redistribute the material, in any medium or format, provided the original work is properly cited. 
loss, weight reduction, decreased lactation, anorexia, irritation, and toxic conditions such as paralysis and allergies (4). The tick control programs have frequently been relied on the utilization of anti-tick chemical compounds, i.e. acaricides. However, the emergence of acaricideresistant tick populations and chemical residuals persistence in animal productions and environment justify the demand for other prevention strategies (5). In this case, vaccination against tick infestation would be an alternative substitute; therefore, it is recommended to study and characterize different tick antigens with likely suitable immunogenicity properties (6).

One of the suggested anti-tick vaccine candidates is the actin-binding tropomyosin (TRM) protein. The molecular structure of this protein includes a coiled-coil dimer which is composed of two parallel-oriented tropomyosin molecules with $\alpha$-helical arrangement (7). This molecule assists in different cell functions, such as intracellular trafficking, cell morphology and cellular contractile force. In invertebrates, several isoforms of tropomyosin have been recognized in muscular and other tissues. Nevertheless, this protein plays a substantial role in immunogenicity and allergenic reactions against ticks and mites so it is an appropriate vaccine candidate against the mentioned parasites $(8,9)$.

Cathepsins (CT) are lysosomal proteases with several members such as cysteine, aspartic, and serine proteases. The physiologic actions of cathepsins are enclosing degradation of intracellular and endocytosis-introduced extracellular proteins. Among them, cysteine proteases in particular are involved in blood degration that is one of the most important biological processes in ticks. Ticks provide their energy for laying eggs, molting, and other related processes from blood digestion. CT $\mathrm{L}$ is one of the members of cysteine protease in Rhipicephalus $(R h)$ microplus which is able to hydrolase tick vitellin and bovine hemoglobin at acidic PH $(10,11)$.

Currently, some polytopic vaccines are evaluated in clinical trials using specific epitopes against infectious agents, cancers, and autoimmune disorders (12-14). In this study, we aimed to predict the characteristics of two genes of Rh.annulatus tick larva as a fused potent vaccine candidates namely CT and TRM, using immunoinformatic tools.

\section{Materials and methods}

Protein sequence retrieval: Since the TRM and CT protein sequences of $R h$. annulatus did not exist in reference databases, the desired sequences of a close species, Rh. microplus TRM, containing 284 amino acid (O97162) and CT containing 332 amino acid (Q9NHB5) were retrieved from UniProtKB database (http://www.uniprot.org/) and saved in FASTA format.

Homology modeling and its validation: The three dimensional (3D) structure of CT and TRM of Rhipicephalus or even other species of ticks was not found in the protein data bank (PDB), so we performed protein modeling. Two most reputable servers in this field are the online servers of the ITASSER (https://zhanglab.ccmb.med.umich.edu/ITASSER/) and SWISS-MODEL (https://swissmodel.expasy.org/). In order to check the potential errors in modeled 3Dstructure, we used Ramachandran plot analysis

at http://mordred.bioc.cam.ac.uk/rapper/ramp age.php.

Secondary structure prediction: Based on PSIPRED server (http://bioinf.cs.ucl.ac.uk/psipred/) and GOR IV server (https://npsaprabi.ibcp.fr/cgi-bin/secpred_gor4.pl), secondary structure was predicted and analyzed.

Prediction of discontinuous B-cell epitopes: Discontinuous B-cell epitopes were predicted from 3D structure of 
proteins epitopes in PDB format by IEDB database

http://tools.iedb.org/main/analysis-tools/.

In order to enhance the reliability, the epitopes were predicted with others bioinformatics servers. After determining B-cell epitopes, we aligned the sequences of predicted epitopes of CT and TRM of the tick with these proteins in cattle using NCBI Blast server for annotating and final selecting of epitopes based on dissimilarity as much as possible.

\section{Prediction of physico-chemical} characteristics: These profiles were illustrated using BcePred servers, IEDB, and ExPAsy-ProtScale (http://www.expasy.org/). The considered factors included hydropathy, availability, flexibility, and exposed surface. The hydrophilic and hydrophobic profiles were estimated on an algorithm introduced by Kyte and Doolittle. (15) Also, surface availability and flexibility of different protein regions were appraised by Emini algorithm and Karplus and Schulz algorithm respectively.

Among the predicted epitopes, those with more availability and the so-called exposed surface were selected using Chimera software. In the end, the construct was investigated in terms of allergenicity using the AllerTOP v. 2.0 server (http://www.ddg-pharmfac.net/AllerTOP/).

The online server SOLpro (http://scratch.proteomics.ics.uci.edu/) was used to investigate Solubility and Antigenicity.

Protein structure analysis: Prosa was used to analyze protein structure at https://prosa.services.came.sbg.ac.at/prosa. php. The overall model quality is shown by the $z$-score. In fact, $z$-score in such models contains the $z$-scores of all protein chains that have been experimentally determined in current PDB. In this plot, different colors are used to distinguish groups of structures from sources (X-ray, NMR).

Construct designing: Sequences of selected epitopes were linked using suitable linker and then the final construct was back translated to DNA at http://web.expasy.org/translate/ and codon of final sequences were optimized in E.coli by jcat online server (expasy/codon optimization tools) (http://www.jcat.de/). Then restriction enzymes (EcoRI/XhoI) were added to $5^{\prime}$ and $3^{\prime}$ ends of sequences and synthetic DNA fragment was cloned into the pET32a. This vector is appropriate for cloning and high-level expression. This is due to the fact that its peptide sequence has been fused with a tag (109aa Trx •Tag thioredoxin protein).

\section{Result}

Selection of epitopes: Finally using BcePred and IEDB servers, two high immunogenic epitopes from each TRM and CT were selected as B-cell epitopes as shown in Table 1. Selection of these epitopes was according to immunoinformatics analysis and all of them had the highest score. To maintain the three-dimensional structure of epitopes, the epitopes of TRM and some amino acids between them were chosen as a larger size epitope.

Table 1. Predicted B-cell epitopes from target antigens.

\begin{tabular}{lll}
\hline Target antigen & Position & Sequence \\
\hline Tropomyosin & $168-188$ & KLAMVEADLERAEERAETGET \\
& $197-222$ & RVVGNNLKSLEVSEEKALQKEETYE \\
Cathepsin & $18-72$ & SQEILRTQWEAFKTTHKKSYQSHMEELLRFKIFTENSLIIAKHNAKYAKGLVSY \\
& $86-142$ & FARIFNGHHGTRKTGGSTFLPPANVNDSSLPKVVDWRKKGAVTPVKDQGQCGSCWA \\
\hline
\end{tabular}

Linker: Since our epitopes are based on B cells and they require a certain degree of flexibility, we used a flexible linker
(GGGGS) to connect epitopes. In the end, the schematic diagram of the construct including epitopes and likers is shown in Figure 1. 
The physico-chemical characteristics:

The molecular weight, number of amino acids, and theoretical P.I of this construct were $19.37,175$, and 7.15 , respectively. The calculated aliphatic index and Grand average of hydropathicity (GRAVY) by Protparam were determined 70.23 and 0.660 respectively.

The total number of negatively charged residues (Asp + Glu) and positively charged residues (Arg + Lys) are the same and equal to twenty-five.

Secondary and Tertiary structures prediction: Predicted secondary structure using online servers PSIPRED was represented in Figure 2. Results obtained from GOR IV server shows that our designed protein was composed of $52.00 \%$ alpha helix $(\mathrm{H}), 8.57 \%$ extended strand and $39.43 \%$ random coil (C) secondary structural elements.

Also, 3D structure of our construct was produced by ITASSER server and is illustrated in Fig 3. C-score is a confidence score for estimating the quality of predicted models by I-TASSER. It is calculated based on the significance of threading template alignments and the convergence parameters of the structure assembly simulations. The normal range of the C-score is -5 to 2 and the higher the $\mathrm{C}$ score value is, the more confident a model will be and vice-versa. The resulted $\mathrm{C}$ score in the present model was 0.14 signifying a high degree of confidence.

A (template modeling) TM-score less than 0.5 shows a correct topology for a model and a TM-score $<0.17$ indicates a random similarity. TM-Score in the model of this paper was $0.73 \pm 0.11$. In addition, rootmean-square deviation (RMSD) for this model was $4.8 \pm 3.1 \AA$.

Validation of tertiary structure and allergenicity prediction: Analysis of the results by Ramachandran plot indicated that number of residues in favored and allowed region were 207 (96.7\%) and 7 ( $3.3 \%$ ) respectively. In addition, there was no amino acid in in outlier region.

The designed construct was determined as non-allergen using AllerTOP v. 2.0 server.

Solubility and Antigenicity of protein:

Obtained results of SOLpro server indicated that this protein is Soluble with the probability of $0.87 \%$ and is Antigenic with the probability of $0.93 \%$.

Protein structure analysis: Based on PSIPRED and GOR IV server results, Zscore of the protein vaccine was -2.51 .

\section{Discussion}

One of the frequent ticks in the world is Rhipicephalus and the species of Rh.annulatus is abundant in north of Iran (16-17). This tick leads to economic losses due to sucking blood and transmission of pathogens such as Anaplasma and Babesia in cattle industry (18-19). Although the chemical control is one of the oldest and the most powerful methods of tick control (20), such method is unendurable, timeconsuming, and costly; thus, attempts are toward designing vaccines based on immunogenic peptides with prediction methods based on amino acid sequence and physico-chemical characteristics (accessibility, flexibility, hydrophobicity, antigenic propensity, exposed surface, turns) (7).

Biological and molecular methods are the most important control methods in recent years (21-22). Attempts have been made to produce vaccine against ticks and some were successful (23-24), but because of the diversity of antigens in species of tick, attempts are still ongoing for producing an effective vaccine (25).

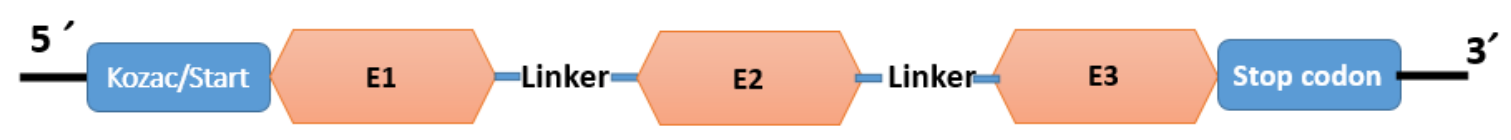


Figure 1. Schematic shape of Final designed CaTro construct. E1: CT epitopes, E2: TRM epitopes, E3: CT epitopes.

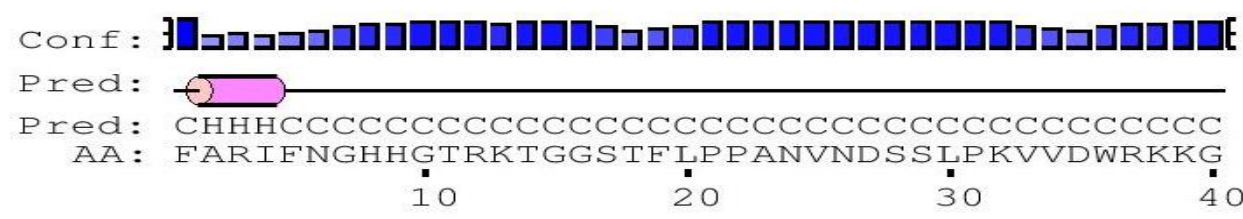

Conf : \}

Pred:

red: CCCCCCCCCCCCCCCCCCCCCHHHHHHHHHHHCCCCCCCC
AA: AVTPVKDQGQCGSCWAGGGGSKLAMVEADIERAEERAETG

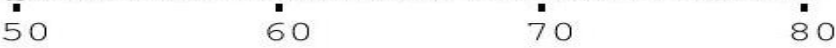

Conf : \} |

Pred: Pred:

red: CCHHHCCCCCCCCCCCCCCCCCCCCCCCCCCCCCCCCCCC
AA: ETKIVELEEELRVVGNNLKSLLEVSEEKALQKEETYEGGGG

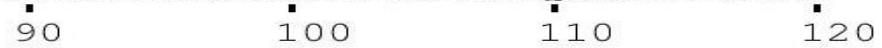

Conf : \}]

Pred: Pred: CCCCCCCCCCCCCCCCEEEECCCCCHHHHHHHHHCCCCHH$^{-}$

AA: SSQEILRTQWEAFKTTHKKȘYQSHMEELLRFKIFTENSLI

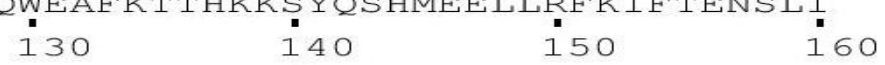

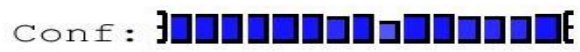

Pred :

AA: IAKHNAKYAKGLVSY

i7o

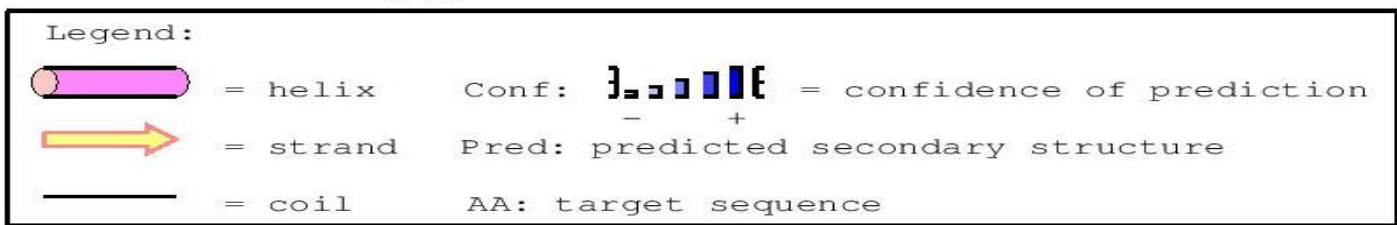

Figure 2. Secondary structure of final Catro construct using online server PSIPRED.

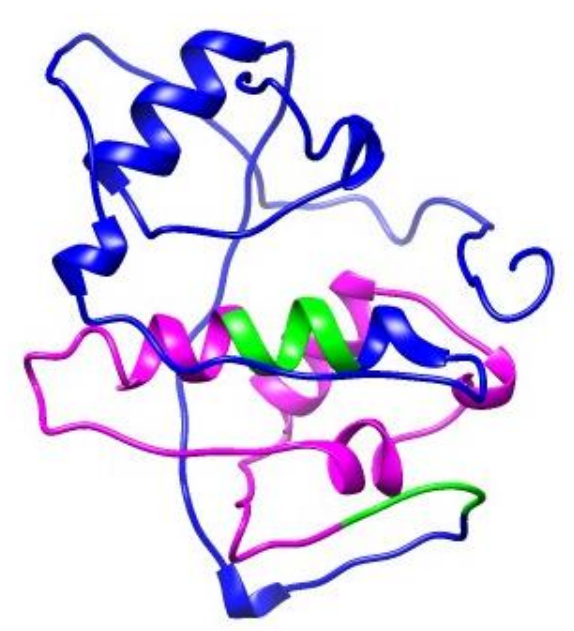


Figure 3. 3D structure of designed construct of CaTro using ITASSER online server by Chimera software; Blue: CT epitope, Pink: TRM, Green: Linkers.

Accordingly, novel strategies are essential to reinforce immune response in cattle and protect them against infestation of tick. Nowadays, in the production of vaccine, allergenicity and concentration of immune response are used from polytopic vaccines due to safety that can produce immune response specifically targeted against the ticks. So far several immunoinformatic researches have been accomplished related to parasitic immunogenic peptides such as TSOL18 in Taenia solium (26), apical membrane protein (AMA-1) in Plasmodium vivax (27), cathepsin Dderived epitope in Schistosoma mansoni (28), EG95 oncosphere protein in Echinococcus granulosus (29), Echinococcus multilocularis (30) and Tand B-cell epitopes of SAG1, GRA2, GRA7 and ROP16 in Toxoplasma gondii (31) and etc.

In this study, we designed a polytopic vaccine from B-cell epitopes of cathepsin and tropomyosin of $R h$. annulatus. The epitopes were chosen by bioinformatics approaches based on high flexibility, hydrophobicity, high accessibility and strong antigenicity. So we used B-cell epitopes to produce an effective vaccine.

Flexible, rigid, and cleavable linkers are three types of experimental linkers for connecting constructed fusion protein. The flexible linkers were composed of small and non-polar amino acids such as Glycine or polar amino acids such as serine or threonine. These features allow them mobility and flexibility. One of the most

common flexible linkers widely used is in the form of GGGGS. In the present study, the mentioned linkers were used to connect the epitopes of B cells (32).

Various evaluations of vaccine properties revealed that our designed vaccine is a highly potential antigen and non-allergen. It should be mentioned that this predicted construct has a high aliphatic index which indicated its stability in a broad range of temperatures.

One of the significant factors in epitope mapping is the presence of proper protein secondary structures such as alpha helix and beta strand, as well as intermediate compositions such as turn and coil. The turn and coil structures have major importance due to more accessibility (33).

\section{Conclusion}

Since, the previous studies shown that CT and TRM are suitable immunogenic proteins as vaccine candidate (34-35), in this study, we designed the potent vaccine candidate of CT and TRM proteins of Rh.annulatus tick larva. Also, we decide to evaluate the protective immunity induced by this vaccine candidate construct against infestation of $R h$. annulatus in rabbits.

\section{Acknowledgments}

We appreciate the Faculty of Veterinary Medicine of Tehran University for financially supporting this research project with code 7506009/6/15.

\section{References}

1. Guglielmone AA, Robbins RG, Apanaskevich DA, Petney TN, Estrada-Peña A, Horak I. The hard ticks of the world. Springer. 2014. P. 978-994. doi: 10.1007/978-94-007-74971

2. Nabian S, Rahbari S, Shayan P, Haddadzadeh H. Current status of tick

fauna in north of Iran. Iran J Parasitol. 2007; 2(1):12-7.

3. Walker JB, Keirans JE, Horak IG. The genus Rhipicephalus (Acari, Ixodidae): a guide to the brown ticks of the world. Cambridge University Press. 2005. P. 2-4. 
4. Cakabay T, Gokdogan O, Kocyigit M. Human otoacariasis: Demographic and clinical outcomes in patients with earcanal ticks and a review of literature. $\mathbf{J}$ Otol. 2016; 11(3):111-17.

5. Roush RT, McKenzie JA. Ecological genetics of insecticide and acaricide resistance. Annu Rev Entomol. 1987; 32:361-80. doi: 10.1146/annurev.en.32.010187.002045

6. Rodríguez MA. Developing Anti-tick Vaccines. Vaccine Design: Methods and Protocols. Vaccines for Veterinary Diseases. 2016. (2)243-259. doi: 10.1007/978-1-4939-3389-1

7. Ranjbar MM, Nabian S, Ahmadi NA, Ghorban K, Sazmand A, Dadmanesh $\mathrm{M}$, et al. Immunoinformatics and similarity analysis of house dust mite tropomyosin. Novelty Biomed. 2015; 3(4):161-70. doi: http://dx.doi.org/10.22037/nbm.v3i4.6 964

8. Gunning PW, Schevzov G, Kee AJ, Hardeman EC. Tropomyosin isoforms: divining rods for actin cytoskeleton function. Trends Cell Biol. 2005;15(6):333-41. doi: 10.1016/j.tcb.2005.04.007

9. Nabian S, Taheri M, Fard RM, Aramoon M. Identification of tropomyosin and its immunological properties from larvae of cattle tick, Boophilus annulatus. Iran J Parasitol. 2013;8(2):242-8.

10. Turk V, Stoka V, Vasiljeva O, Renko M, Sun T, Turk B, et al. Cysteine cathepsins: from structure, function and regulation to new frontiers. Biochim Biophys Acta. 2012;1824(1):68-88. doi: 10.1016/j.bbapap.2011.10.002

11. Franta Z, Frantová H, Konvičková J, Horn M, Sojka D, Mareš M, et al. Dynamics of digestive proteolytic system during blood feeding of the hard tick Ixodes ricinus. Parasit Vectors. 2010;3:119. doi: 10.1186/1756-3305-3-119.
12. Nisbet A, Huntley J, Mackellar A, Sparks N, McDevitt R. A house dust mite allergen homologue from poultry red mite Dermanyssus gallinae (De Geer). Parasite Immunol. 2006; 28(8):401-5. doi: 10.1111/j.13653024.2006.00862.x.

13. Sabhnani L, Manocha M, Sridevi K, Shashikiran D, Rayanade R, Rao DN. Developing subunit immunogens using $\mathrm{B}$ and $\mathrm{T}$ cell epitopes and their constructs derived from the F1 antigen of Yersinia pestis using novel delivery vehicles. FEMS Immunol Med Microbiol. 2003; 38(3):215-29.

14. Seib KL, Dougan G, Rappuoli R. The key role of genomics in modern vaccine and drug design for emerging infectious diseases. PLoS Genet. 2009; 5(10):e1000612.

doi: 10.1371/journal.pgen.1000612

15. Kyte J, Doolittle RF. A simple method for displaying the hydropathic character of a protein. J Mol Biol. 1982; 157(1): 105-32.

16. Razmi GR, Glinsharifodini M, Sarvi S. Prevalence of ixodid ticks on cattle in Mazandaran province, Iran. Korean $\mathbf{J}$ Parasitol. 2007; 45(4): 307-10.

17. Nabian S, Taheri M, Ranjbar MM, Sazmand A, Youssefy P, Nazaralipour GR. Assessment and partial purification of serine protease inhibitors from Rhipicephalus (Boophilus) annulatus larvae. Rev Bras Parasitol Vet. 2014; 23( 2): 187-93.

18. Taheri M, Nabian S, Ranjbar M, Mazaheri NFR, Gerami SA, Sazmand A. Study of vitellogenin in Boophilus annulatus tick larvae and its immunological aspects; Trop Biomed. 2014; 31(3): 398-405.

19. McGowan MJ, Homer JT, O Dell GV, Mcnew RW, Barker RW. Performance of ticks fed on rabbits inoculated with extracts derived from homogenized ticks, Anaplasma maculatum Koch (Acarina: Ixodidae). J Parasitol. 1980; 66: 42-48. 
20. Parizi LF, Pohl PC, Masuda A, Vaz Ida S Jr. New approaches toward antiRhipicephalus (Boophilus) microplus tick vaccine. Rev Bras Parasitol Vet. 2009; 18(1):1-7.

21. Guerrero FD, Andreotti R, Bendele KG, Cunha RC, Miller RJ, Yeater K, et al. Rhipicephalus (Boophilus) microplus aquaporin as an effective vaccine antigen to protect against cattle tick infestations. Parasit Vectors. 2014;7:475. doi: 10.1186/s13071-0140475-9. doi: 10.1186/s13071-0140475-9

22. Rodriguez M, Penichet ML, Mouris AE, Labarta V, Lorenzo LL, Rubiera $\mathrm{R}$, et al. Control of B. microplus populations in grazing cattle vaccinated with a recombinant $\mathrm{Bm} 86$ antigen preparation. Vet Parasitol. 1995;57(4):339-49.

23. Willadsen P, Bird P, Cobon GS, Hungerford J. Commercialization of a recombinant vaccine against Boophilus microplus. Parasitology. 1995;110 Suppl:S43-50.

24. De la Fuente J, Rodriguez M, GarciaGarcia JC. Immunological control of ticks through vaccination with Boophilus microplus gut antigens. Ann N Y Acad Sci. 2000;916:617-21.

25. De Vos S, Zeinstra L, Taoufik O, Willadsen P, Jongejan F. Evidence for the utility of the Bm86 antigen from Boophilus microplus in vaccination against other tick species. Exp Appl Acarol. 2001;25(3):245-61.

26. Zimic M, Gutiérrez AH, Gilman RH, López C, Quiliano M, Evangelista W, et al. Immunoinformatics prediction of linear epitopes from Taenia solium TSOL18. Bioinformation. 2011; 6(7):271-4. doi.10.6026/97320630006271

27. Bueno LL, Lobo FP, Morais CG, Mourão LC, de Ávila RAM, Soares IS, et al. Identification of a highly antigenic linear B cell epitope within Plasmodium vivax apical membrane antigen 1 (AMA-1). PLoS One. 2011;6(6):e21289.

doi:

10.1371/journal.pone.0021289.

28. Fuaad AAH, Roubille R, Pearson MS, Pickering DA, Loukas AC, Skwarczynski M, et al. The use of a conformational cathepsin D-derived epitope for vaccine development against Schistosoma mansoni. Bioorg Med Chem. 2015 Mar 15;23(6):130712. doi: 10.1016/j.bmc.2015.01.033

29. Woollard DJ, Gauci CG, Heath DD, Lightowlers MW. Protection against hydatid disease induced with the EG95 vaccine is associated with conformational epitopes. Vaccine. 2000; 19(4):498-507.

30. Gomase VS, Chitlange NR. Prediction of MHC class antigen peptides from Echinococcus multilocularis: Application of computer intelligence. Scientific Reports. 2012; 1(3):191. doi.org/10.4172/scientificreports.191

31. Cao A, Liua Y, Wangb J, Lic X, Wanga S, Zhaoa Q, et al. Toxoplasma gondii: Vaccination with a DNA vaccine encoding $\mathrm{T}$ - and B-cell epitopes of SAG1, GRA2, GRA7 and ROP16 elicits protection against acute toxoplasmosis in mice. Vaccine. 2015; 27; 33(48):6757-62. doi: 10.1016/j.vaccine.2015.10.077.

32. Chen X, Zaro J, Shen WC. Fusion protein linkers: Property, design and functionality; Adv Drug Deliv Rev. 2013; 65(10): 1357-69. doi:10.1016/j.addr.2012.09.039.

33. Kaur H, Raghava G. An evaluation of $\beta$-turn prediction methods. Bioinformatics. 2002; 18(11):1508-14.

34. Saidi S, Nabian S, Ebrahimzade E, Najafi A, Moosazadeh Moghaddam M, Sazmand A, et al. Identification and characterization of a cathepsin L-like cysteine protease from Rhipicephalus (Boophilus) annulatus; Exp Appl Acarol. Doi: 10.1007/s10493-0159993-1. 2015

35. Nikpay A, Nabian S, Taheri M. Analysis of Immunogenic Relevant Proteins in Rhipicephalus (Boophilus) 
annulatus Tick. J Arthropod Borne

Dis. 2012;6(1):36-44. 